\title{
CONSIDERAÇÕES CLÍNICAS E ETIOLÓGICAS DAS SÍNDROMES EPILÉPTICAS SINTOMÁTICAS COM PAROXISMOS OCCIPITAIS BLOQUEADOS À ABERTURA DOS OLHOS
}

\author{
Gilson Edmar Gonçalves e Silva', Maria Eunice de Vasconcelos Xavier Coelho², \\ Silvia Gomes Laurentino ${ }^{3}$
}

\begin{abstract}
RESUMO - A síndrome da epilepsia parcial com paroxismos occipitais no eletrencefalograma (EEG) mostra características clínicas heterogêneas. Visando descrever esta rara forma de epilepsia, investigamos a significância dos achados eletrofisiológicos e clínicos em oito pacientes com paroxismos occipitais bloqueados pela abertura dos olhos. Os pacientes foram submetidos a exames radiológicos de RNM do encéfalo e/ou TC de crânio. Houve concordância entre as alterações do EEG e do tipo de crise em 5 pacientes, e entre as anormalidades anatômicas na TC ou RNM com anormalidades focais no EEG em 7 casos. Nossos resultados confirmam que este padrão inusitado origina-se de uma expressão eletrográfica multifatorial.
\end{abstract}

PALAVRAS-CHAVE: paroxismo occipital, crises occipitais, síndromes epilépticas occipitais.

\begin{abstract}
Clinical and ethiological considerations about the symptomatic epileptic syndromes with occipital paroxism bloqued when the eyes open

ABSTRACT - The syndrome of idiophatic partial epilepsy with occipital paroxysms in the EEG shows a considerable clinical heterogeneity. The present paper investigated the signficance of electrophysiologic and clinical characteristics in eigth patients with occipital paroxysms blocked by the eye opening. All patients were submitted to radiological exams including brain MRI and/or CT. There was agreement between EEG findings and type of seizures in 5 patients and between anatomical abnormalities in the MRI or CT and EEG focal abnormalities in 7 patients. Our results confirm that the unusual pattern comes from eletrographic multifactors origins.
\end{abstract}

KEY WORDS: occipital paroxysms, occipital seizures, occipital epileptic syndromes.

Os primeiros casos de paroxismos epilépticos occipitais, bloqueados à abertura dos olhos foram definitivamente descritos, em 1982, por Gastaut ${ }^{1}$ ao relatar uma síndrome epiléptica benigna, que ocorre em crianças e é caracterizada, clinicamente, por crises visuais e episódios de "enxaqueca-like". Ainda na década de 80 , e começo dos anos 90 , foram descritos alguns raros casos, inicialmente confundidos com uma forma atípica da síndrome de Sturge-Weber $^{2,3}$, porém com características próprias: uma associação peculiar de paroxismos no eletroencefalograma (EEG) interictal (descargas contínuas de ponta-onda na região occipital, uni ou bilateralmente, bloqueadas à abertura dos olhos), de crises epilépticas (visuais, tipo enxaqueca, parciais simples e complexas, seguida ou não de generalização secun- dária), calcificações occipitais bilaterais e doença celíaca ${ }^{4-6}$. Entretanto, essa nova e rara síndrome epiléptica foi melhor caracterizada por autores italianos (Magoudda et al.) que estudaram 12 pacientes, constituindo a maior casuística sobre o tema7. Posteriormente, outras publicações surgiram, porém com um número pequeno de $\operatorname{casos}^{8,9}$. Até o final da década de 90 eram menos de 70 casos descritos, num levantamento feito por um de nós ${ }^{10}$. Na maioria dos casos descritos haveria a associação com a doença celíaca, porém, mais raros eram os pacientes que não tinham esta patologia ${ }^{11}$, inclusivena nossa publicação, que se caracterizou como o primeiro caso publicado na literatura nacional.

Entretanto, ao continuarmos as nossas pesquisas sobre o assunto, encontramos pacientes com as

Programa de Pós-Graduação (PG) em Neuropsiquiatria, Universidade Federal de Pernambuco (UFPE) - Centro de Ciências da Saúde (CCS), Recife PE, Brasil. 'Professor Adjunto Doutor de Neurologia, Diretor do CCS/UFPE; ${ }^{2}$ Professora Adjunta de Neurologia Infantil, UFPE; 3Mestranda do Programa de PG em Neuropsiquiatria.

Recebido 25 Setembro 2001, recebido na forma final 12 Novembro 2001. Aceito 21 Novembro 2001.

Dr. Gilson Edmar Gonçalves e Silva - Rua Muniz Tavares 147/601 - 52050-170 Recife PE - Brasil. 
características clínicas e eletrencefalográficas descritas nestas síndromes epiléticas, porém com várias outras causas etiológicas, diferentes das calcificações da doença celíaca. O objetivo deste artigo é contribuirmos para uma melhor elucidação desta peculiar expressão eletrencefalográfica.

\section{MÉTODO}

Foram estudados 8 pacientes oriundos do Ambulatório de Epilepsia do Hospital das Clínicas da Universidade Federal de Pernambuco, de um dos Ambulatónios do SUSeda Clínica Privada. Ospacientesselecionados para o presente estudo eram 5 do sexo masculino e 3 do feminino. A idade, na ocasião da primeira consulta, variou de 2 a 30 anos (média 16,3 anos).

Foi estabelecido, como critério de inclusão dos pacientes, o registro no EEG, em vigília, de pontasoccipitais, uni ou bilateralmente, bloqueadas à abertura dos olhos, além da história clínica de crises epilépticas visuais e de outrostipos, e de estudo anatômico, através da neuroimagem.

A Tabela 1 contém a identificação dos pacientes. Consta da Tabela 2 o tipo de crises epilépticas relatadas pelos pacientes ou seus familiares, a droga antiepiléptica em uso (DAE) e os resultados dos EEG.

\section{RESULTADOS}

Todos os nossos pacientes apresentavam, como queixa, crises epilépticas do tipo visual, cujos relatos mais frequentes eram: ora a visão de manchas, ora a de formas geométricas (círculos ou quadrados), sempre coloridos. Apenas um dos 8, não tinha queixa de crise visual, explicável pela idade muito precoce do aparecimento dos sintomas ( 2 anos); porém, apresentava, ao exame neurológico, baixa visual e
Tabela 1. Dados de identificação.

\begin{tabular}{lcccc}
\hline Caso & Paciente & Registro & Sexo & Idade (anos) \\
\hline 1 & M.J.S.A & HC: $1247755-4$ & F & 17 \\
2 & A.G.C & HC: $1111027-4$ & M & 30 \\
3 & A.P & HC: $1100251-0$ & F & 22 \\
4 & A.M.S. & HC: $1000088-2$ & F & 21 \\
5 & P.V.S.S. & HC: $1183978-9$ & M & 02 \\
6 & R.F.A. & SUS: 150873 & M & 12 \\
7 & R.L.R.S. & Cl. Privada & M & 17 \\
8 & R.S.S. & Cl. Privada & M & 10 \\
\hline
\end{tabular}

F, Feminino; $M$, Masculino.

nistagmo óptico.

As crises visuais eram associadas a crises parciais complexas, com generalização secundária em 2 pacientes, a apenas crises parciais motoras em 3 deles, a crises parciais motoras, com generalização secundária em 1 e apenas a generalização secundária em 1 caso. O paciente de 2 anos de idade (Caso 5), que não tinha queixa de crise visual, apresentava crise de desvio conjugado dos olhos para a direita, seguida de perda da consciência.

Houve concordância entre a lateralidade na descrição da crise focal e o resultado do EEG em 5 pacientes (Casos 1, 3, 4, 6, 8). Em 2 (Casos 2 e 7) não haviam dados clínicos de lateralidade e em apenas 1 paciente (Caso 5) não havia concordância entre a lateralidade da crise e o EEG.

Constam da Tabela 3 os resultados do EEG e dos exames de neuroimagem (TC/RM) dos nossos pacientes.

Tabela 2. Dados clínicos.

\begin{tabular}{llll}
\hline Caso no & Tipo de crises & DAE & EEG \\
\hline 1 & Visual + CPC+TCG & FB+DPH & P. Occip. bilateral \\
2 & Visual + CPC+TCG & FB+CBZ & P. Occip. à D \\
3 & Visual + parcial motora D & DFH & P. Occip. bilateral mais à E \\
4 & Visual + adversiva D & CBZ & P. Occip. à E \\
5 & Desvio dos olhos para D + perda da consciência & CBZ & P. Occip. Bilateral \\
6 & Visual + adversiva D & CBZ & P. Occip. à E \\
7 & Visual + TCG & CBZ & P. Occip. à D \\
8 & Visual + adversiva E + TCG & CBZ & P. Occip. bilateral mais à D \\
\hline
\end{tabular}

CPC, crise parcial complexa; TCG, crise tônico-clônica generalizada; FB, fenobarbital; DPH, difenil-hidandoina; CBZ, carbamazepina; P. Occip, ponta occipital; E, esquerda; D, direita. 
Tabela 3. Exames complementares.

\begin{tabular}{|c|c|c|}
\hline Caso no & EEG & $\mathrm{TC} / \mathrm{RM}$ \\
\hline 1 & P. Occip. bilateral & $\begin{array}{l}\text { Calcificação occip. } \\
\text { bilateral }\end{array}$ \\
\hline 2 & P. Occip. à D & $\begin{array}{l}\text { Calcificação occip. } \\
\text { à D }\end{array}$ \\
\hline 3 & P. Occip. bilateral mais à E & $\begin{array}{l}\text { Lesão atrófica } \\
\text { occip. E }\end{array}$ \\
\hline 4 & P. Occip. à E & $\begin{array}{l}\text { Área hipodensa } \\
\text { occip. E }\end{array}$ \\
\hline 5 & P. Occip. Bilateral & $\begin{array}{l}\text { Área hipodensa } \\
\text { occip. bilateral }\end{array}$ \\
\hline 6 & P. Occip. à E & Atrofia occip. E \\
\hline 7 & P. Occip. à D & $\begin{array}{l}\text { Anomalia vascular } \\
\text { à D }\end{array}$ \\
\hline 8 & P. Occip. bilateral mais à D & Normal \\
\hline
\end{tabular}

Dos nossos 8 pacientes selecionados, 7 deles apresentavam alterações nos exames de imagem em concordância com os achados do EEG, e em apenas 10 exame de neuroimagem foi normal.

\section{DISCUSSÃO}

O lobo occipital pode ser sede de descargas epileptiformes (pontas ou complexos ponta-onda), bloqueadas ou não à abertura dos olhos. Estas descargas, em sua maioria, constituem um dos elementos das síndromes epilépticas do lobo occipital. Porém, podem ser de baixa epileptogenicidade, surgindo em crianças sem crises, mas relacionadas à cegueira congênita e sugerindo um processo de deaferentação ${ }^{12}$.

As síndromes epilépticas do lobo occipital podem ser classificadas em idiopáticas e sintomáticas. Entre as síndromes epilépticas idiopáticas, estão as epilepsias parciais benignas da infância, com paroxis-

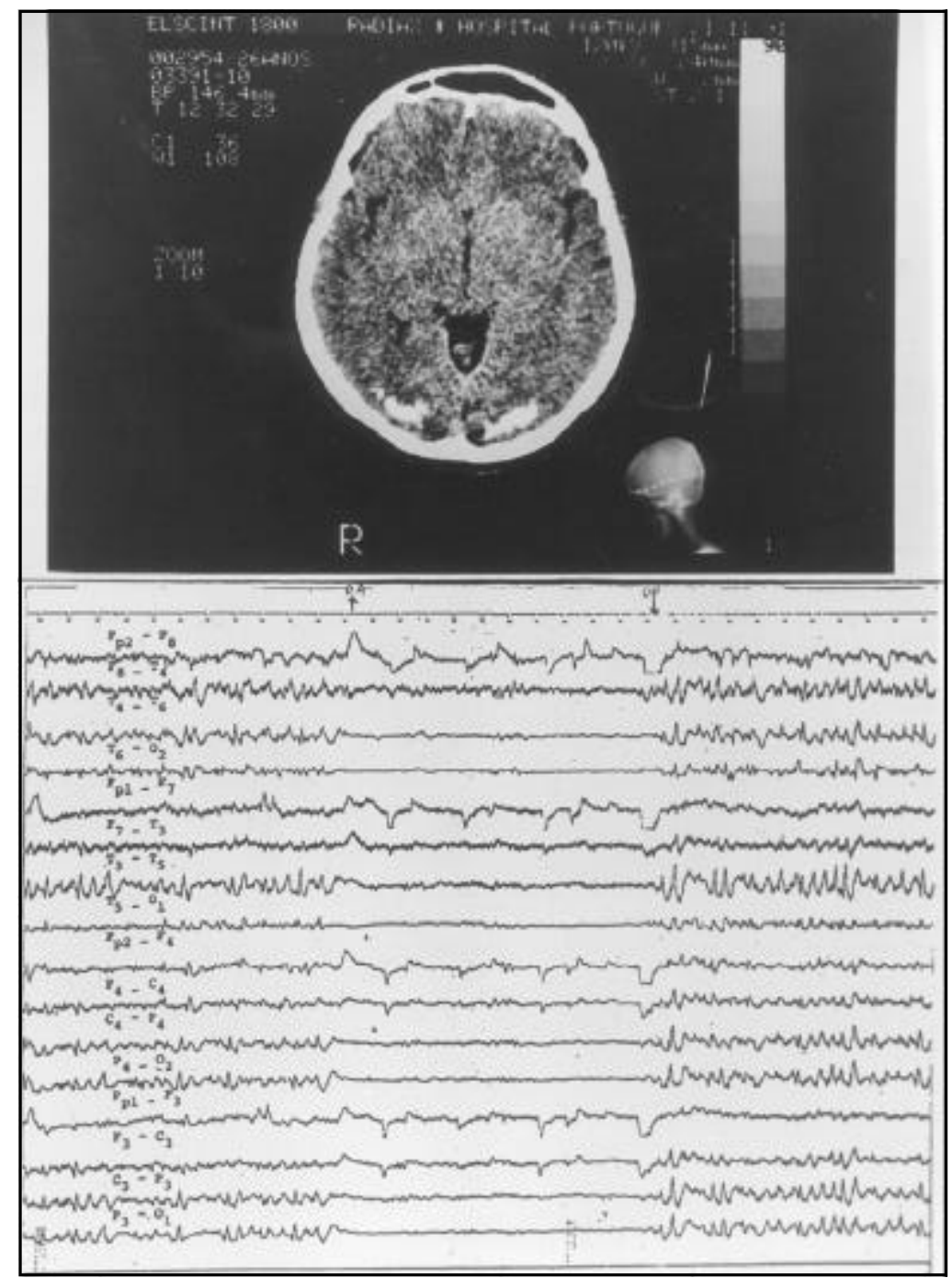

Fig 1. EEG e tomografia do Caso 1: calcificações occipitais bilaterais. 


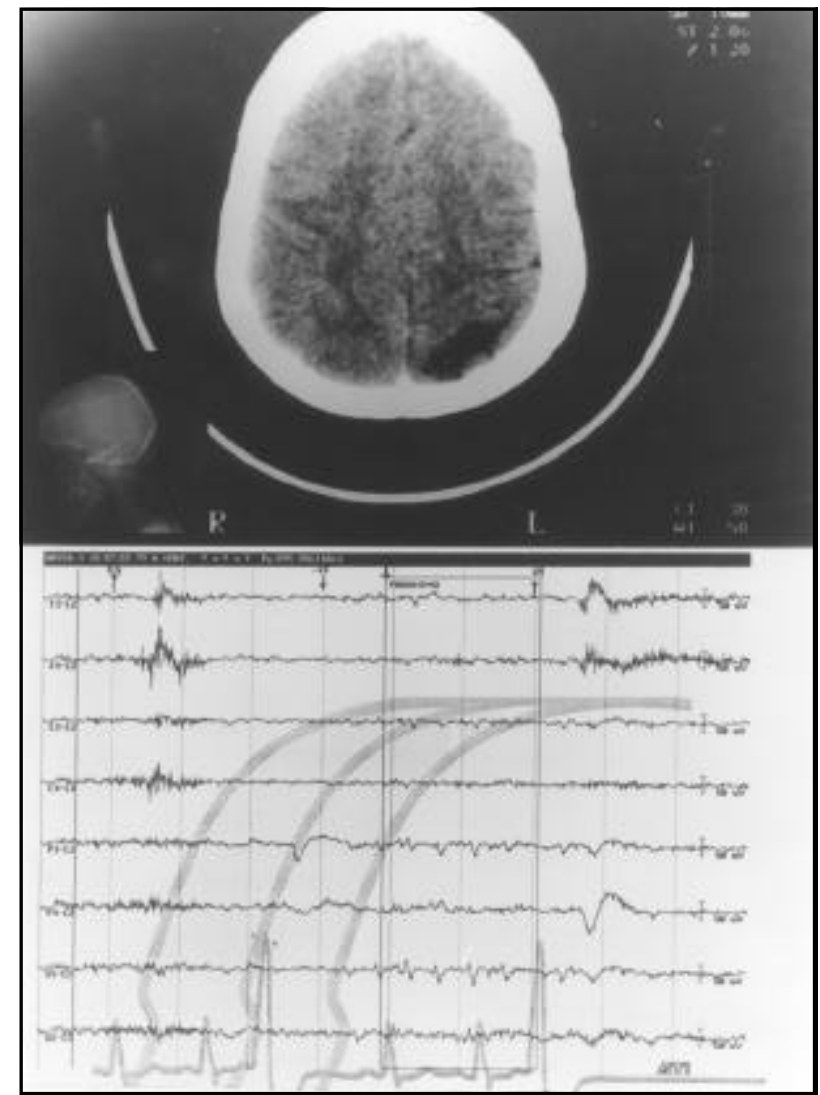

Fig 2. EEG e tomografia do Caso 4: área hipodensa occipital esquerda.

mos occipitais (EPBI-O) ${ }^{13}$. Foi Gastaut ${ }^{1}$ quem primeiro descreveu este quadro clínico, em 1982, constituído de crises visuais, acompanhadas de crises hemiclônicas, parciais complexas ou tônico-clônicasgeneralizadas; como manifestação pós-ictal havia cefaléia e vômitos; os paroxismos occipitais são de elevada amplitude, bilaterais, quase rítmicos, bloqueados à abertura dos olhos, sendo o ritmo de base normal; o diagnóstico diferencial se faz com as epilepsias sintomáticas do lobo occipital (mais raras na infância), com as epilepsias occipitais fotossensíveis e com as enxaquecas basilares ${ }^{13,14}$. Uma variante desta síndrome foi descrita por Panayotopoulus (in Costa et al. ${ }^{13}$ ), caracterizada por vômitos ictais, desvio óculo-cefálico, início em idade mais precoce, predomina durante o sono e o EEG evidencia pontas occipitais, não reativas à abertura dos olhos, quando realizado em vigília5.

Já as síndromes epilépticas occipitais sintomáticas são mais raras e caracterizadas por crises visuais elementares (luzes, cores) ou como manifestações alucinatórias mais elaboradas ${ }^{13}$. O EEG é frequentemente anormal, ocorrendo descargas epileptiformes com projeção occipital, mas também têmporo-

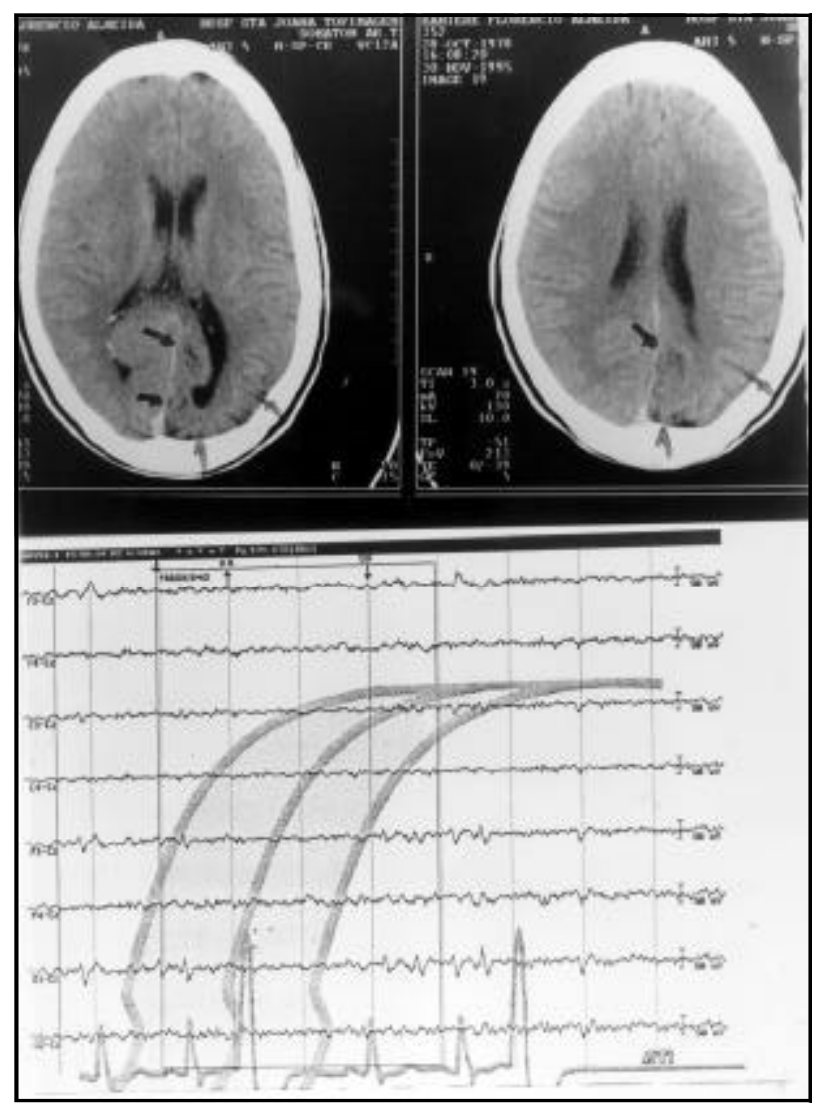

Fig 3. EEG e tomografia do Caso 6: atrofia occipital esquerda.

occipital ou parieto-occipital ${ }^{15}$; esses paroxismos, em geral, não são bloqueados à abertura dos olhos ${ }^{14}$. Entretanto, o bloqueio desses paroxismos não está necessariamenteassociado a uma forma benigna de epilepsia, podendo estar associado a várias condições clínicas, com paroxismos não bloqueados à abertura dos olhos ${ }^{16}$.

Todos os nossos pacientes apresentaram, no EEG, os paroxismos occipitais (pontas ou complexos ponta-onda), uni ou bilateralmente, bloqueados à abertura dos olhos (critério de inclusão). Do ponto de vista clínico, os pacientes apresentavam crises do tipo visual, associadas a crises parciais motoras simples, mais frequentes, ou a parciais complexas, com ou sem generalização secundária. A única criança do grupo não tinha referências, na história clinica, a crises visuais, pois a sua baixa idade impede a descrição destes sintomas. Em 5 dos nossos casos havia concordância entre o tipo de crise e o resultado do EEG.

Outro dado que merece destaque é que, dos 8 casos selecionados, 7 deles apresentaram anormalidade no exame de neuroimagem: calcificações occipitais em 2 casos (Fig 1), lesão atrófica occipital em 
2 casos (Fig 2), área hipodensa occipital em 2 casos (Fig 3) e anomalia vascular occipital em 1 caso. Um paciente apresentou TC e RM normais, mesmo tendo o EEG com paroxismo lateralizado. Todos os 7 casos, nos quais foram encontrados anormalidades estruturais nos exames de neuroimagem, apresentavam concordância topográfica com as anormalidades encontradas no EEG.

Em conclusão, o presente estudo não permite tirar conclusões definitivas, pelo pequeno número de casos. Porém, justifica a sua análise o fato desta situação eletroclínica ser bastante rara. Algumas reflexões, entretanto, devem ser feitas com base nos nossos resultados: 1 - 0 achado eletrográfico dos paroxismos occipitais bloqueados à abertura dos olhos não é só característico das epilepsias occipitais benignas; 2 - Esta anormalidade eletrográfica surge também nas epilepsias occipitais sintomáticas. Enfim, este peculiar paroxismo nos parece uma expressão eletrográfica, de origem multifatorial.

\section{REFERÊNCIAS}

1. Gastaut H. A new type of epilepsy: benign partial epilepsy of childhood with occipital spikes-waves. Eletroenceph Clin Neurophysiol 1982; 13:13-22.

2. Ambrosetto P, Ambrosetto G, Michelucci R, Bacci A. Sturge-Weber syndrome without port-wine facial nevus (report of 2 cases estudied by CT). Child's Brain 1983;10:387-392.
3. Garwicz S, Mortensson W. Intracranial calcification mimicking the Sturge-Weber syndrome (a consequence of cerebral folic acid deficiency?) Pediat Radiol 1996;5:5-9.

4. Zaniboni MG, Ambrosetto P, Lambertini A, et al. Epilepsia, calcificazioni endocraniche, malattia celiac: un'associazione forse non più casuale. Minerva Ped 1991;43:215-218.

5. Gobbi G, Sorrenti G, Santucci M, et al. Epilepsy with bilateral occipital calcification: a benign onset with progressive severity. Neurology 1988;38:913-920.

6. Molteni N, Bardella MT, Baldassari AR, Bianchi PA. Celiac disease associated with epilepsy and intracranial calcifications: report of two patients. Am J Gastroenterol 1988;88:992-994.

7. Magaudda A, Dalla Bernardina B, DeMarco P, Sfaello Z, Longo M Bilateral occipital calcification, epilepsy and coeliac disease: clinical and neuroimaging features of a new syndrome. J Neurol Neurosurg Psychiatry 1993;56:885-889.

8. Cronin CC, Jackson LM, Feighery C, Shanaran N. Coeliac disease and epilepsy. Q J M 1998;91:303-308.

9. Ferroir JP, Ferrelon G, Billy C, Huon R, Herry JP. Epilepsy, cerebral calcification and celiac disease. Rev Neurol 1997;153:354-356.

10. Gonçalves e Silva GE. Síndrome epiléptica associada a calcificações occipitais bilaterais, sem doença celíaca. Neurobiologia 1999;62:61-64.

11. Sammaritano M, Andermann F, Melanson D, Guberman A, Tinuper P, Gastaut $\mathrm{H}$. The syndrome of intractable epilepsy, bilateral occipital calcifications and folic acid deficiency. Neurology 1988;38:239.

12. Wada JA, Ellingson RJ. Clinical neurophysiology of epilepsy. In Handbook of electroencephalography and clinical neurophysiology Revised series vol 4. Amsterdam: Elsevier 1990:237-310.

13. Costa JC, Palmini A, Yacubian EMT, Cavalheiro EA. Fundamentos neurobiológicos das epilepsias: aspectos clínicos e cirúrgicos. São Paulo: Lemos Editorial 1998:163-242.

14. Roger J, Dravet C, Bureau M, Dreifuss FE, Wolf P. Les syndromes epileptiques de l'enfant et de l'adolescent. London. John Libbey Eurotex. 1984:163-174.

15. Guerreiro CAM, Guerreiro MM. Epilepsia. 2.Ed . São Paulo: Lemos Editorial, 1996:50

16. Fonseca LC, Tedrus GMAS. Atividade epileptiforme occipital com ou sem bloqueio pela abertura dos olhos. Arq Neuropsiquiat 1995;53:724729. 